\title{
The Evolution of Scanned Probe Microscopy: Two Decades of Rapidly Evolving Techniques and Applications
}

\author{
P.E. Russell ${ }^{*}$, M.E. Salmon ${ }^{*}$ \\ *AIF - North Carolina State University, Campus Box 7531, Raleigh, NC 27695, USA
}

Scanned Probe Microscopy (SPM) includes a range of microscopy techniques which are generally thought of as evolutionary to the scanning tunneling microscope (STM) first reported by Binnig and Rohrer [1]. The SPM technique in most common use currently is generally referred to as Atomic Force Microscopy (AFM); and its popularity stems primarily from the wide range of materials for which it is suitable for high resolution imaging, rather than the ultimate resolution of the technique. In fact, the SPM technique of STM is generally agreed to provide the highest spatial resolution of any SPM technique, and yet it is certainly not the most commonly utilized. The origins of STM concepts and technology were presented by the Field Ion Microscopist R. Young of the National Bureau of Standards (now NIST) and summarized in the JULY 1975 issue of Physics today [2]. It was several years later Bining and Roher, at IBM Zurich, extended the work of Young to obtain the first demonstration of atomically resolved imaging features using a physically scanned metal probe and a semiconductor surface in vacuum.

In contrast to the development of electron and ion microscopies, scanned probe microcopy, of which STM was the original, started out with atomic resolution capability (Figure 1a). The timing of the early STM developments in the early 1980's which was also the time of rapid development of personal computers and low cost, high quality instrumentation, image processing and display electronics, was ideal for the very rapid evolution of the instrumentation and technique. Not only did STM advance rapidly from a complex, ultrahigh vacuum surface physics specialty to a low cost commercially available non-vacuum microscopy, but closely related techniques quickly began to emerge. The first of these was AFM, which quickly opened non-conducting materials to the scanned probe microscope. Many forms of scanned force microscopy (SFM) have developed since the original in 1986 and now atomic resolution micrographs using AFM are easily obtainable (Figure 1b).

SPM as a family of techniques is possibly the most versatile of all microscopy techniques that have atomic resolution capabilities. Due to the flexibility of SPM instrumentation, the investigation of a variety of materials properties such as modulus, electronic and magnetic properties, thermal stability, and surface chemistry are all possible by specific techniques often due to customization of the probes used or the environment the samples are imaged under. SPM has the added ability to controllably manipulate materials with atomic level precision. For instance, scientists at IBM have provided strong evidence to support the existence of Quantum Chaos Theory by creating atomic corrals of $\mathrm{Fe}$ on $\mathrm{Cu}[3]$. The ability to perform in-situ imaging within sample growth environments is very important for understanding the creation of nanostructures such as the self-assembled nanofibers seen in Figure 2. SPM is unique in that the resolution of the instrument does not dependant on vacuum. Imaging environments include vacuum, air, or liquid, and does not require lenses, focus, nor stigmation. One extreme example of environmental flexibility is the design and 
fabrication of an ultra-compact SPM for the analysis of dust and soil particles on Mars to be included on the NASA Phoenix mission planned for 2007 [4].

[1] - Binning and Rohrer, Physics Today, Aug. (1986).

[2] - R. Young, Physics Today, Z4, 42 (1971).

[3] - M.F. Crommie, C.P. Lutz, D.M. Eigler, Science 262, 218-220 (1993).

[4] - http://phoenix.lpl.arizona.edu/

[5] - Highlights for Children, September (1991).
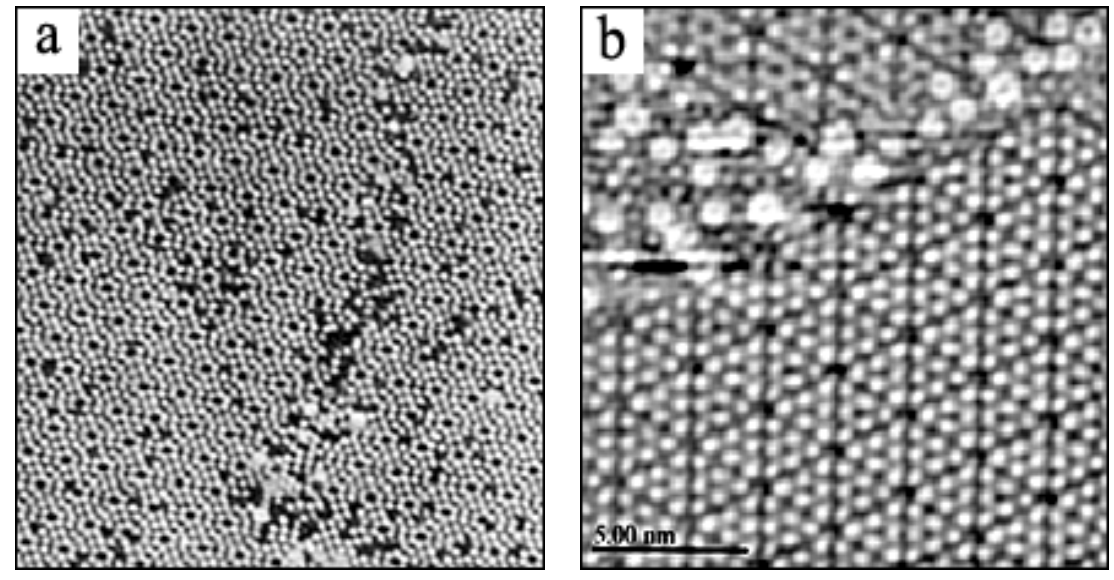

Figure 1. (a) 47nm x 47nm UHV-STM micrograph of $<111>$ Si $7 x 7$ surface reconstruction (b) $15 \mathrm{~nm} \times 15 \mathrm{~nm}$ Non-contact UHV-AFM height image of $<111>\mathrm{Si}$. Both images courtesy of JEOL.

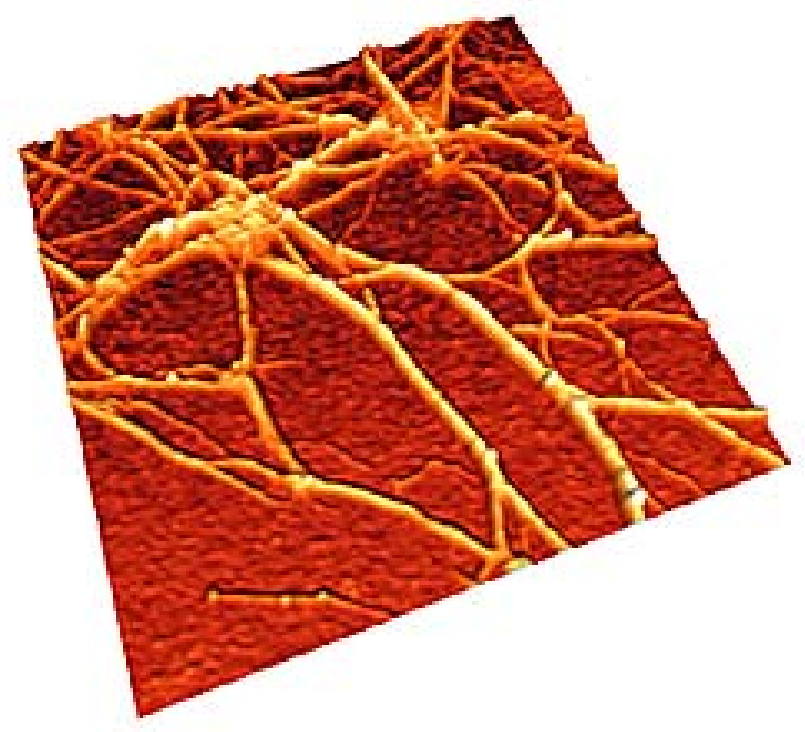

Figure 2. $1 \mu \mathrm{m} \times 1 \mu \mathrm{m}$ TappingMode ${ }^{\mathrm{TM}}$ height image of self-assembled nanofibrous network grown on $\mathrm{Si}_{3} \mathrm{~N}_{4}$ from corrosion of $\mathrm{Al}$ metal in $0.1 \mathrm{wt} \%$ Metylphosphonic acid solution. 